\title{
Self-Assembly of Semiconducting-Plasmonic Gold Nanoparticles with Enhanced Optical Property for Photoacoustic Imaging and Photothermal Therapy
}

\author{
Zhen Yang1, 2, Jibin Song ${ }^{2}$, Yunlu Dai², Jingyi Chen ${ }^{3}$, Feng Wang 3 , Lisen Lin², Yijing Liu², Fuwu Zhang², \\ Guocan Yu², Zijian Zhou², Wenpei Fan², Wei Huang1, Quli Fan ${ }^{1 凶}$ and Xiaoyuan Chen² ${ }^{\varpi}$ \\ 1. Key Laboratory for Organic Electronics and Information Displays \& Institute of Advanced Materials (IAM), Jiangsu National Synergetic Innovation Center \\ for Advanced Materials (SICAM), Nanjing University of Posts \& Telecommunications, Nanjing 210023, China; \\ 2. Laboratory of Molecular Imaging and Nanomedicine (LOMIN), National Institute of Biomedical Imaging and Bioengineering (NIBIB), National Institutes of \\ Health (NIH) Bethesda, MD 20892, USA; \\ 3. Department of Chemistry and Biochemistry, University of Arkansas, Fayetteville, Arkansas 72701, USA. \\ $\triangle$ Corresponding authors: Quli Fan, Email: iamqlfan@njupt.edu.cn; Jibin Song, Email: jibin.song@nih.gov; Xiaoyuan Chen. Email: shawn.chen@nih.gov \\ (c) Ivyspring International Publisher. This is an open access article distributed under the terms of the Creative Commons Attribution (CC BY-NC) license \\ (https://creativecommons.org/licenses/by-nc/4.0/). See http://ivyspring.com/terms for full terms and conditions.
}

Received: 2017.04.12; Accepted: 2017.05.01; Published: 2017.06.01

\begin{abstract}
Although various noble metal and semiconducting molecules have been developed as photoacoustic (PA) agents, the use of semiconducting polymer-metal nanoparticle hybrid materials to enhance PA signal has not been explored. A novel semiconducting-plasmonic nanovesicle was fabricated by self-assembly of semiconducting poly(perylene diimide) (PPDI) and poly(ethylene glycol (PEG) tethered gold nanoparticles (Au@PPDI/PEG). A highly localized and strongly enhanced electromagnetic (EM) field is distributed between adjacent gold nanoparticles in the vesicular shell, where the absorbing collapsed PPDI is present. Significantly, the EM field in turn enhances the light absorption efficiency of PPDI, leading to a much greater photothermal effect and a stronger photoacoustic signal compared to PDI nanoparticle or gold nanovesicle alone. The optical property of the hybrid vesicle can be further tailored by controlling the ratio of PPDI and gold nanoparticle as well as the adjustable interparticle distance of gold nanoparticles localized in the vesicular shell. In vivo imaging and therapeutic evaluation demonstrated that the hybrid vesicle is an excellent probe for cancer theranostics.
\end{abstract}

Key words: perylene diimide, gold nanoparticle, vesicle, semiconducting-plasmonic coupling, photoacoustic imaging.

\section{Introduction}

With the rapid development of photoacoustic (PA) imaging technology, more sensitive and efficient PA contrast agents are urgently needed to provide sufficiently high contrast-to-noise ratio (CNR) to enable high-resolution PA imaging.[1-5] To acquire a PA image of tumor with high CNR, the near infrared (NIR) PA probes should exhibit strong NIR absorption and high photothermal conversion efficiency.[6-8] Semiconducting molecules including small molecules and polymers, such as perylene diimide (PDI) $[9,10]$ and polypyrrole [11], have been widely investigated as PA and photothermal agents, due to their high light absorption efficiency and absorption spectra in the NIR region.[12, 13] In comparison with commonly studied organic PA probes such as indocyanine green and methylene blue, and inorganic PA probes such as carbon nanotube (CNT) and reduced graphene oxide (rGO), etc.,[14-16] semiconducting molecules exhibit stronger absorption in the NIR region, higher photothermal conversion efficiency, outstanding stability against harsh environments,[17] and lower incidence of biological side effects. $[9,18]$ More importantly, PDI semiconducting polymer in nanoparticle form can 
further increase its light absorption efficiency by several orders of magnitude when compared to the dispersed molecules, leading to greatly enhanced PA signal.[9] We have previously demonstrated that the PDI nanoparticles could be used as a PA contrast agent for bioimaging of tumor and vital organs, such as the mouse brain.[9]

Plasmonic metal nanoparticles with unique optical properties known as localized surface plasmon resonance (LSPR) are of considerable interest for its use in bioimaging, biosensing, and surface enhanced Raman spectroscopy.[19-23] The electromagnetic (EM) field generated from the metal surface can be greatly amplified by decreased distances of interparticle junctions in plasmonic assemblies, which are known as "hot spots".[24-28] We previously reported that, plasmonic coupling between gold nanoparticles (AuNPs) can enhance the light absorption efficiency of the carbon nanomaterials, such as CNT and rGO, leading to enhanced efficiency in PA and photothermal properties.[29-31] Plasmonic vesicles assembled from AuNPs also exhibit strong EM field between nanoparticles due to the interparticle plasmonic coupling, which allows effective PA imaging and SERS sensing.[32] In another example, rGO was encapsulated in the plasmonic vesicle cavity to greatly enhance its PA signal.[31] More recently, Le Ru. et al. reported that the shift and broaden of the small molecular resonances when it was absorbed on metal nanoparticle surface, revealing the enhanced optical absorption of the organic dyes (such as Crystal Violet, Rhodamine 6G and Nile Blue) in contact with the metal nanocrystals.[33] However, little is known about the effects of the EM field arising from plasmonic coupling on the photothermal and PA effects of semiconducting molecules/polymers.[34, 35]

Herein, we report a novel semiconductingplasmonic vesicle assembled from amphiphilic gold nanoparticles grafted with high density poly(perylene diimide) (PPDI) and poly(ethylene glycol) (PEG) (Au@PPDI/PEG) (Figure 1). The hybrid nanovesicle with tuneable size and LSPR could be facilely obtained by self-assembly of Au@PPDI/PEG through our previously reported thin film-rehydration method.[36, 37] As shown in Figure 1, in the hybrid vesicle, collapsed hydrophobic PPDI and AuNPs form the vesicular shell, where PPDI is localized between adjacent AuNPs via п-п stacking. The plasmonic coupling between AuNPs in the vesicular structure red shifts the maximum absorption to $700 \mathrm{~nm}$, which is co-localized with the absorption maximum of the PPDI shell. Upon NIR laser irradiation, interparticle plasmonic coupling of AuNPs not only increases the light absorption efficiency of the AuNPs, but also serves as local nanoantennae to enhance the optical energy absorption of the PPDI shell at its absorption maximum.[36, 37] As a result, the PA signal of the $\mathrm{Au} @ P P D I / P E G$ vesicle is about 3.5-fold higher than that of a mixture of PDI NPs and Au vesicles. The enhancement of PA signal of the hybrid vesicle was confirmed by simulating the optical spectra and near-field distribution with the discrete dipole approximation (DDA) using the DDSCAT 7.3 program. In vivo experimental results demonstrated that the hybrid vesicle is an excellent candidate as both PA imaging probe and photothermal agent for cancer theranostics. $[30,38]$

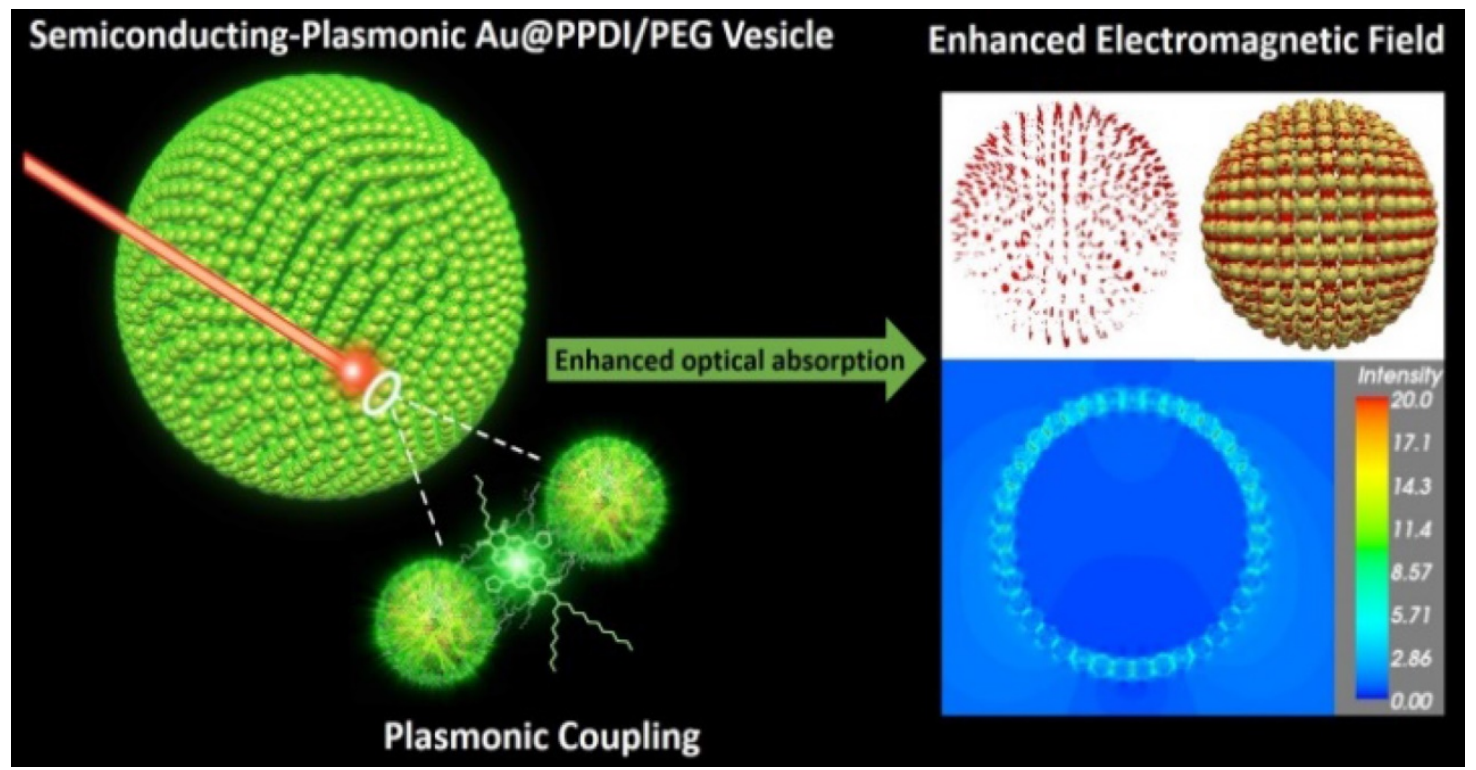

Figure 1. Schematic illustration of the semiconducting-plasmonic vesicle of gold nanoparticle coated with PPDI and PEG. The strong electromagnetic field in the vesicular shell is due to interparticle plasmonic coupling, leading to enhanced optical property. 


\section{Results and discussion}

To prepare hybrid semiconducting-plasmonic vesicles, the PPDI with thiol end group was first synthesized. PDI monomer was synthesized by introducing two pyrrolidines in its bay position to promote NIR absorption, which shows an asymmetric structure with a long alkyl chain and amine for exchange with the polymer precursor (Scheme S1, Supporting Information). The successful synthesis of amine functionalized PDI monomer was confirmed by $1 \mathrm{H}$ NMR, 13C NMR, and MALDI-TOF (Figure S1-S9, Supporting Information). To synthesize non-conjugated polymer containing sufficient PDI pendants, post-polymerization modification method was introduced for polymerization of PDI (Scheme S2, Supporting Information). Poly(pentafluorophenyl acrylate) (PPFPMA) was synthesized as a precursor to conjugate PDI (Figure S10-11, Supporting Information). The copolymer precursor $(\mathrm{Mn}=10.9$ $\mathrm{KDa})$ was synthesized with styrene and PPFPMA monomer acting as an activated ester by atom transfer radical polymerization (ATRP) with 2,2'-dithiobis[1(2-bromo-2-methylpropionyloxy)]ethane (DTBE) as an initiator. This disulfide derivative can form covalent Au-S bonds to conjugate with the AuNP surface. For the copolymer precursor, PPFPMA can be replaced by functional groups containing amine, thus amine based PDI pendants linked to the non-conjugated copolymer precursor can be formed by transesterification between amine PDI and PPFPMA in THF by refluxing for three days. This yields a replacement efficiency of up to $90 \%$. The obtained PPDI has $\sim 25$ PDIs in one polymer chain, calculated from PDI to PS ratio (5:8), and molecular weight $(\mathrm{Mn}=33.1 \mathrm{KDa})$ of the polymer is derived through 1H NMR and GPC data (Figure S12 and S13, Supporting Information).

Amphiphilic semiconducting-plasmonic Au@PPDI/PEG was further prepared by mixing citric acid capped AuNP ( $15 \mathrm{~nm}$ in diameter) and the mixture of PDI and PEG in DMF. Three different kinds of Au@PDDI/PEG with PPDI to PEG ratios of 1:1, 2:1, and 3:1 were prepared, which were calculated by ${ }^{1} \mathrm{H}$ NMR (Figure S14, Supporting Information). For the Au@PPDI/PEG with PPDI to PEG ratios of 3:1, the number of PPDI and PEG attached onto AuNP are 150 and 50, respectively, calculated by TGA results of Au@PPDI/PEG (Figure S15, Supporting Information), resulting in 3750 PDI molecules per AuNP. Successful attachment of PPDI onto AuNPs was verified by comparing the UV-vis spectra of the vesicle to the AuNP and PDI polymer (Figure 2a). The UV-vis spectrum of PDI has two spectral features with the fingerprint band at $700 \mathrm{~nm}$ and the vibronic progression peak at $650 \mathrm{~nm}$. In the presence of strong $\Pi-\Pi$ stacking among PDIs, the intensity of the vibronic progression peak at $650 \mathrm{~nm}$ will be stronger than that of the fingerprint band at $700 \mathrm{~nm}$. Thus, the relative intensity of the absorption peaks at 650 and $700 \mathrm{~nm}$ in UV-vis spectra can be used to monitor the extent of п-п stacking. As shown in Figure 2a, Au@PPDI/PEG exhibited significantly enhanced absorption intensity at $650 \mathrm{~nm}$ relative to PDI polymer alone and the broadened peak from the mixture of PDI and AuNPs, presumably due to effective п-п interactions between PPDIs on the AuNP surface. Moreover, $\mathrm{Au} @$ PPDI/PEG were purple colored, which was different from PPDI with green colour and AuNPs with red colour, suggesting that PPDIs were attached to AuNPs (Figure 2b).

The hybrid vesicles were first prepared by self-assembly of Au@PPDI/PEG with the PPDI to PEG ratios of 3:1 using the thin film-rehydration method as we previously reported.[36, 37] The contrast difference between the interior and the shell in the transmission electron microscopy (TEM) image of the vesicles confirmed the characteristically hollow nanostructures (Figure 2c). SEM image showed the morphology of vesicles and closely assembled AuNPs in the vesicular shell, further confirming the hollow structure (Figure 2d). Dynamic light scattering (DLS) profiles reveal that the size of obtained hybrid vesicle is $95 \pm 10 \mathrm{~nm}$ (Figure S16, Supporting Information). The close attachment of AuNPs in the vesicular shell leads to strong interparticle plasmonic coupling, as evidenced by the significant red-shifted spectral profile and broadened absorption peaks (Figure 2a). The as-prepared hybrid vesicles showed brown colour due to the coupling between the AuNPs (Figure 2b).[31] The LSPR of the vesicle of Au@PPDI/PEG with PPDI to PEG ratio of 3 matched well with the absorption peak of PPDI at $700 \mathrm{~nm}$.

The photothermal effect and PA performance of $\mathrm{Au} @ P P D I / P E G$ vesicles illuminated with a $700 \mathrm{~nm}$ laser were further examined. As control experiments, we used PMMA without absorption in the NIR region to replace PPDI and prepared Au@PMMA/PEG nanovesicles. PDI nanoparticles were also prepared (see supporting information for preparation method). Upon NIR laser irradiation, the hybrid vesicle exhibited an enhanced photothermal effect when compared to the mixture of PDI NP and Au@PMMA/PEG vesicles (Figure S17, Supporting Information). Furthermore, PA signal of the hybrid vesicle is higher than the mixture, as displayed in Figure 3a, b. The PA amplitudes of all the samples in aqueous solution increased linearly with increasing optical density at $700 \mathrm{~nm}\left(\mathrm{OD}_{700}\right)$ (Figure 3c). At $\mathrm{OD}_{700}$ $=1$, the PA intensity of AuNP@PPDI/PEG vesicles 
was over 3.5 times higher than that observed in the mixture of PDI NPs and Au@PMMA/PEG vesicles, about 2.5 times higher than that observed for $\mathrm{Au} @ \mathrm{PMMA} / \mathrm{PEG}$ vesicles, and over 10 times higher
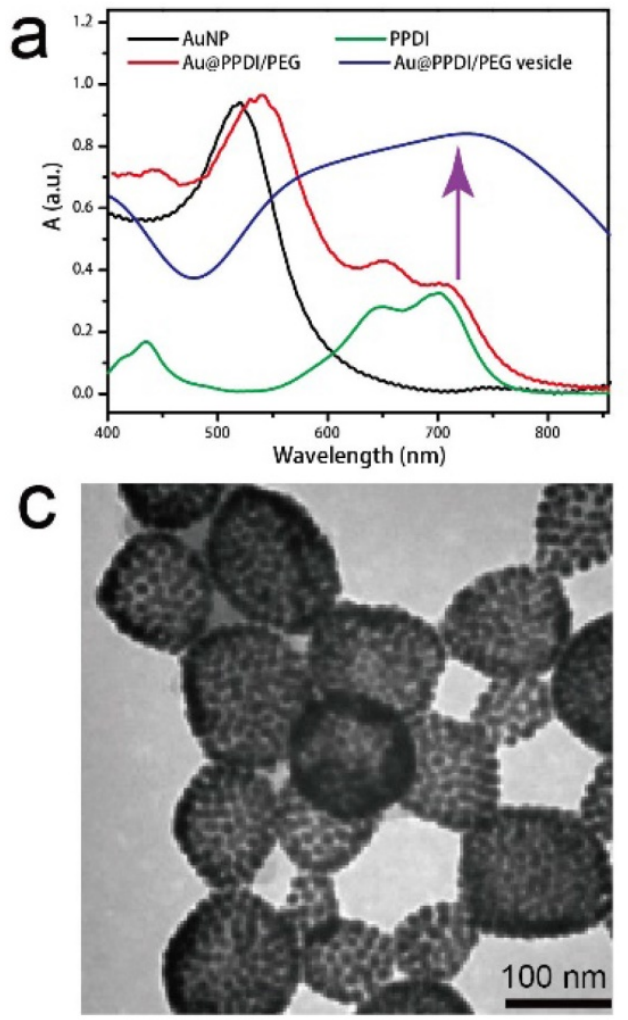

than the PDI NPs, as shown in Figure 3d. The strong enhancement was a result of EM hotspots in $\mathrm{Au} @$ PPDI/PEG vesicles.
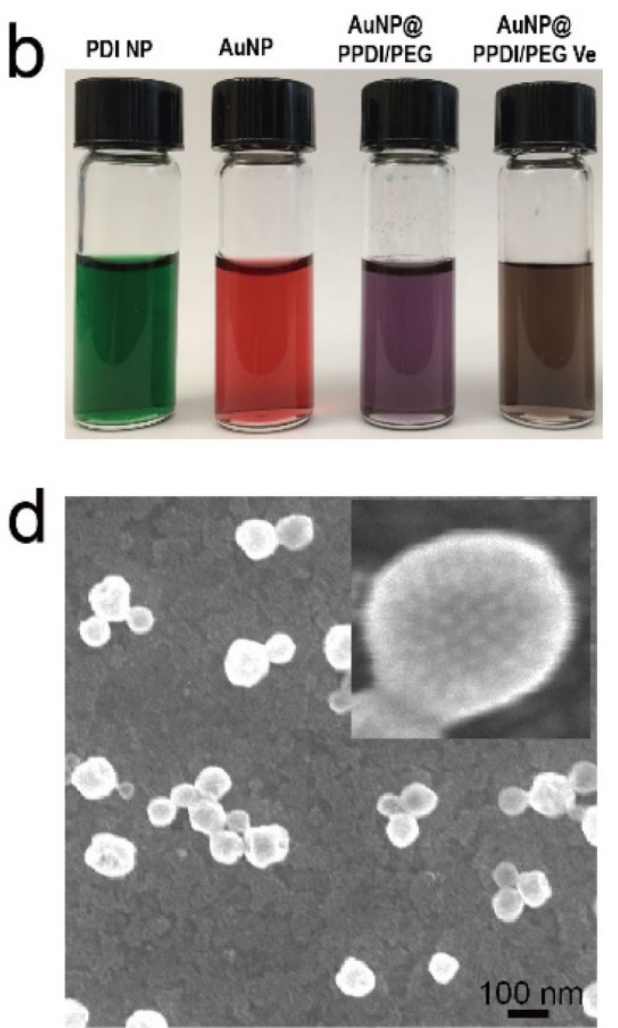

Figure 2. (a) UV-vis spectra of the Au NPs in water, PPDI and Au@PPDI/PEG in chloroform, and the Au@PPDI/PEG vesicles in water. (b) Photographs of the samples in water. TEM (c) and SEM (d) images of Au@PPDI/PEG vesicles.
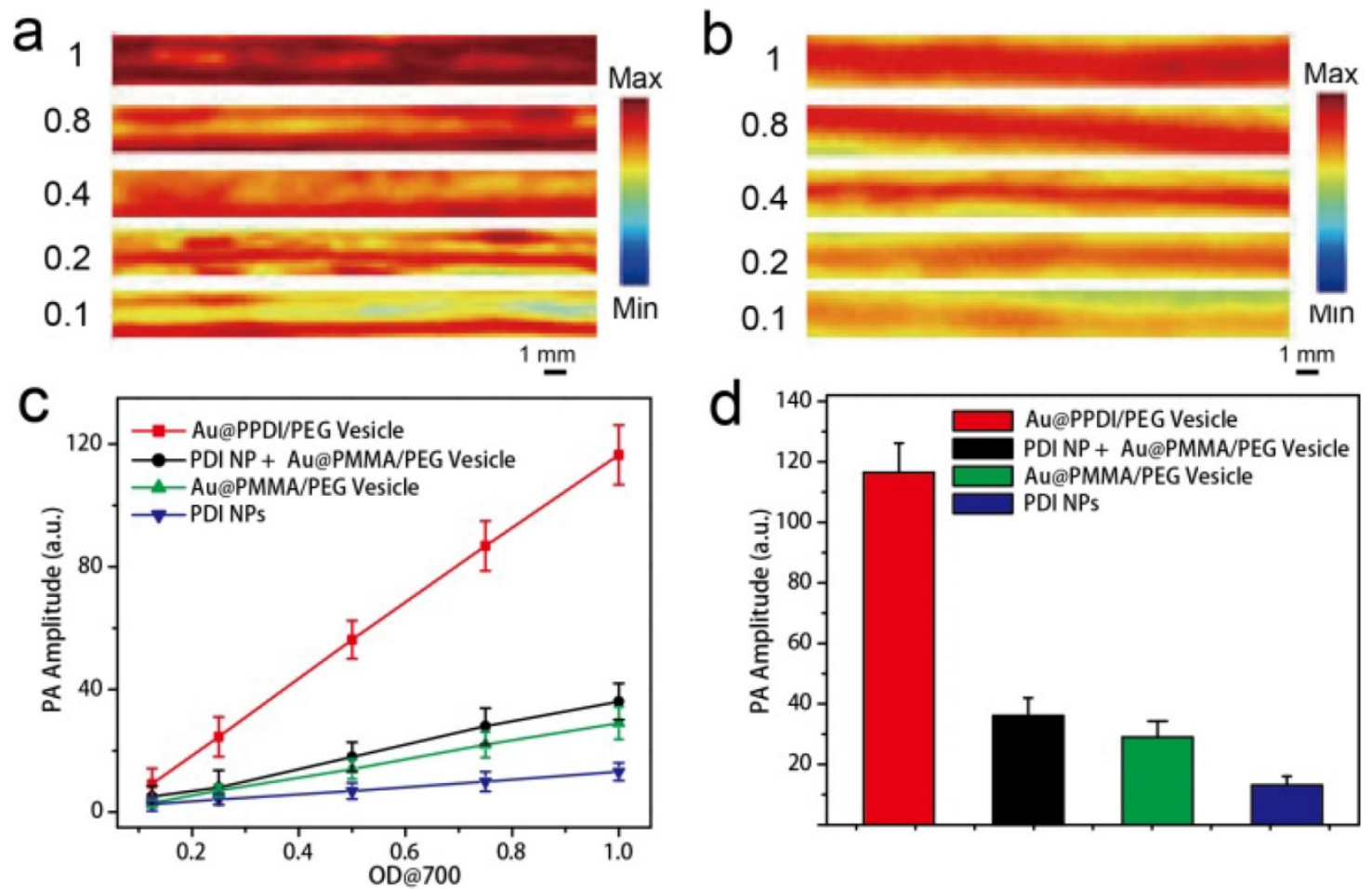

Figure 3. PA images of (a) Au@PPDI/PEG vesicle and (b) the mixture of Au@PMMA/PEGvesicles and PDI NPs aqueous solutions, (c) PA amplitudes of the samples in aqueous solution as a function of $\mathrm{OD}_{700}$ value. (d) PA amplitudes of the samples at the $\mathrm{OD}_{700}$ value is 1.0 . 
To systematically investigate the relationship between the intensity of EM field and PA signal enhancement, we further prepared three types of $\mathrm{Au} @$ PPDI/PEG vesicles with average interparticle distances of about 1, 2, and $3 \mathrm{~nm}$ by self-assembly of $\mathrm{Au} @$ PPDI/PEG with different PPDI to PEG ratios of 1:1, 2:1 and 3:1, respectively. From the TEM images (Figure 4a-c), we can clearly observe and measure the interparticle distance between AuNPs of the three kinds of vesicles, which increased with increasing ratio of PPDI to PEG attached on AuNP surface. The maximum absorption peak of the vesicle red shifted with decreasing ratio of PPDI to PEG (Figure 4d). PA signal of the hybrid vesicles decreased with increasing interparticle distance, due to the reduced plasmonic coupling and EM field (Figure 4e).[20, 39, 40] The strongest PA signal of the hybrid vesicle was observed when the interparticle distance was $\sim 1 \mathrm{~nm}$, which is consistent with the greatest EM filed in the 1 nm gap of nanogapped AuNPs.[21, 41]

The optical spectra and near-field distribution of the hybrid vesicles were further calculated under the DDA method using the DDSCAT 7.3 program.[30] The vesicle is composed of 501 solid Au nanospheres with a diameter of $15 \mathrm{~nm}$ (Figure 5a). The vesicle has a diameter of $200 \mathrm{~nm}$ and is hollow inside. The distance between the nanosphere ranges from 0.7 to $1.0 \mathrm{~nm}$.
The overall target is composed of 301647 dipole moments. The AuNPs are modelled by the complex dielectric response function of bulk $\mathrm{Au}$ and the vesicle assembly is submerged in a continuous medium with a dielectric constant of 1.33, which corresponds to liquid water.

The optical cross sections were averaged over two orthogonal polarization directions of the incident light. The optical efficiency, $Q$, is reported as the ratio of the respective optical cross section to $\Pi \cdot a_{a f f}^{2}$, where the effective radius, $a_{\text {eff, }}$ is defined as the radius of a sphere whose volume is the same as the 501 AuNPs. The vesicle has a broad extinction peak at $\sim 640 \mathrm{~nm}$, which is the sum of a relatively strong absorption peak at $\sim 610 \mathrm{~nm}$ and a weak, but very broad band across 600 to $900 \mathrm{~nm}$ due to scattering. The "hot spots" (Figure 5b,c) and near-field distribution (Figure $5 \mathrm{~d}$ ) of the vesicle was calculated at the incident wavelength of $700 \mathrm{~nm}$. Figure 5b,c shows the distribution of hot spots, which is defined as places with near-field enhancement of a factor of five or larger relative to the incident. The near-field intensity of the vesicle in the $x-y$ plane was plotted in Figure 5e. Between these assembled AuNPs in the vesicle, the field enhancement could be as much as 20 times larger than that of the incident.

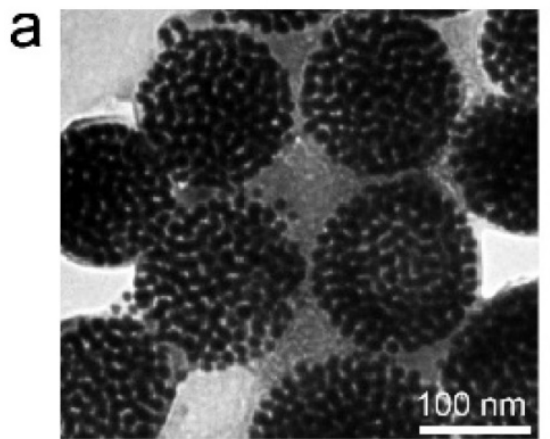

d

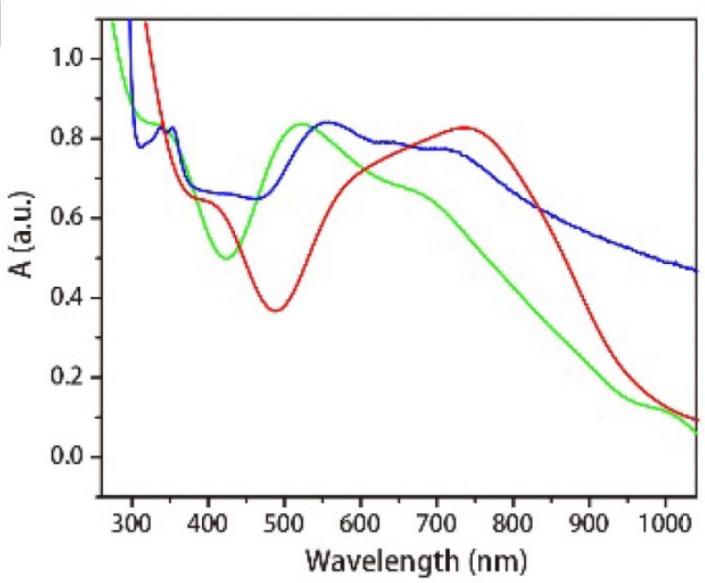

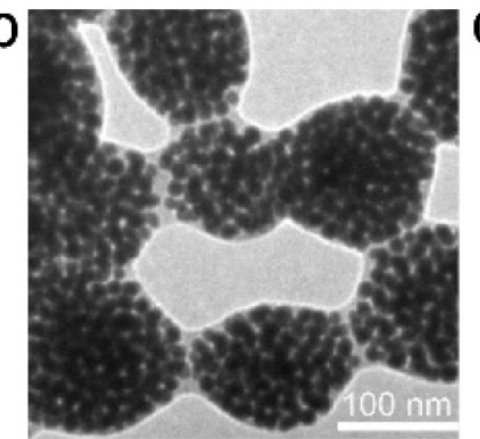
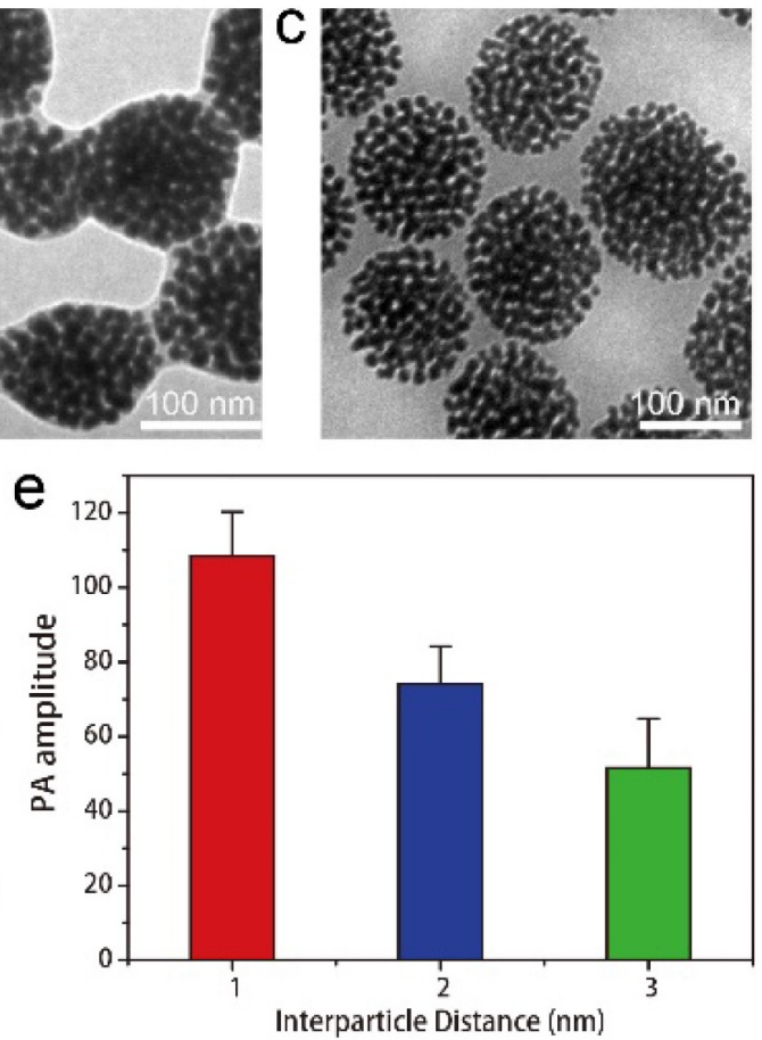

Figure 4. TEM images of the Au@PPDI/PEG vesicles with the interparticle distances of $\sim 1 \mathrm{~nm}$ (a), $2 \mathrm{~nm}$ (b) and 3 nm (c). (d) UV-vis spectra of the Au@PPDI/PEG vesicles with the interparticle distances of $1 \mathrm{~nm}$ (red line), $2 \mathrm{~nm}$ (blue line) and 3:1 (green line). (e) PA amplitude of the vesicle with different interparticle distance. 

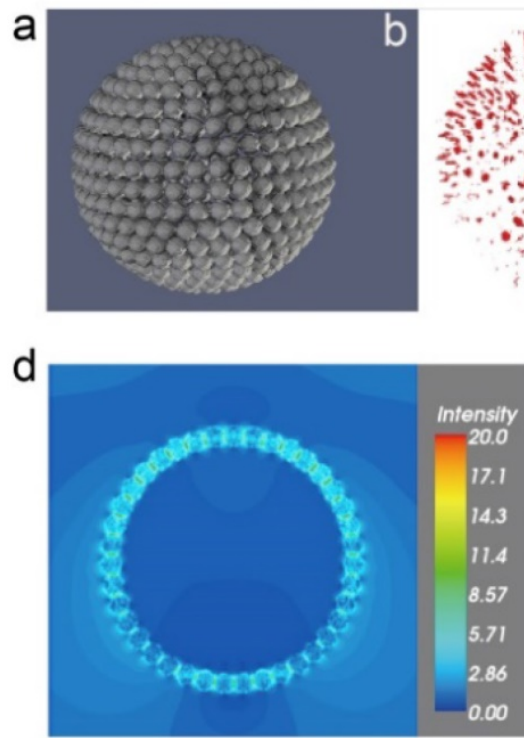
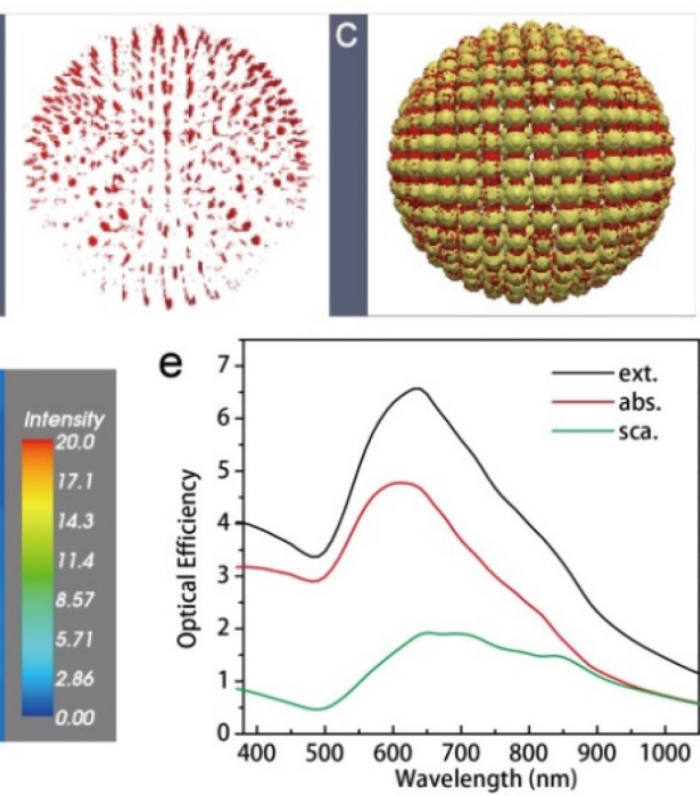

Figure 5. Optical efficiency and near-field distribution of the vesicle calculated using the discrete dipole approximation. A 3D model of the vesicle (a), representation of the hot spots (red) (b) and overlaid image (c) with local field enhancement of a factor of five or larger at the incident wavelength of $700 \mathrm{~nm}$. The Au nanospheres are shown in yellow. (d) Near-field distribution in a $x-y$ cross-section close to the center of the vesicle at the incident wavelength of 700 nm. Only electric field strength is shown. (e) The optical efficiency of the vesicle shown in its extinction (black), absorption (red), and scattering (blue) spectra.

PA imaging, as an emerging imaging approach, enables multiscale high special and deep resolution imaging of tissue and biological structures. Encouraged by the enhanced PA signal of the $\mathrm{Au} @$ PPDI/PEG vesicles, we investigated the capability of the vesicles as a theranostic agent by using U87MG tumor-bearing mice. $200 \mu \mathrm{L}$ of vesicles in PBS $(0.5 \mathrm{mg} / \mathrm{mL})$ were intravenously injected into mice with tumor volume about $\sim 60 \mathrm{~mm} 3$ for PA images, which was recorded over time by pulsed laser excitation at $700 \mathrm{~nm}$. Compared with preinjection, the PA signal in the tumor region increased over time, and reached maximum plateau at $\sim 30 \mathrm{~h}$ postinjection (Figure 6a, b). The strong 3D PA signal showed even distribution of the vesicles in the tumor, which is consistent with the ultrasound image (Figure 6a). As a control experiment, Au@PMMA/PEG vesicles with a similar size to hybrid vesicles were also tested. At $24 \mathrm{~h}$ postinjection, the intensity in the tumor region of the mice treated with Au@PPDI/PEG vesicles was 2.2 times higher than that of the Au@PMMA/PEG vesicles (Figure 6b). These results suggest that Au@PPDI/PEG vesicles can act as an excellent PA imaging probe for $3 \mathrm{D}$ signal reconstruction of the tumor, which clearly delineates that the PA signals are detectable both inside and outside of the blood vessels of tumor, and provides detailed information in tumor sections, such as size, shape and extent of the neovascularization (Figure 6a). [42, 43] The PA spectra of the vesicles in the tumor region are similar to the absorption spectra of the sample in solution (Figure $6 c)$.
We further employed the hybrid vesicle for in vivo photothermal therapy (PTT). Consistent with the high tumor accumulation and photothermal effect of the hybrid vesicles, excellent PTT effect was observed in the tumor area as indicated by the infrared image of the tumor-bearing mice (Figure 7a). After laser irradiation of the tumor region at $30 \mathrm{~h}$ postinjection, the tumor temperature reached $80^{\circ} \mathrm{C}$ (Figure $7 \mathrm{~b}$ ) after irradiation with NIR lasr at $0.3 \mathrm{~W} / \mathrm{cm}^{2}$ for $5 \mathrm{~min}$, which is sufficient to kill all cancer cells. However, the average temperature of the tumor treated with the mixture of PDI NPs and Au@PEG/PMMA vesicles only reached $\sim 50{ }^{\circ} \mathrm{C}$. Tumor growth was completely suppressed in the group treated with Au@PPDI/PEG vesicle and laser irradiation, in contrast with continued tumor growth in the control groups (Figure 7c). The novel hybrid vesicles were capable of highly efficient hyperthermia in the tumor region using laser at a very low power density. Hematoxylin and eosin (H\&E) staining was further employed to study the tumor tissues through pathological examination (Figure $7 \mathrm{~d}$ ). The cancer cells of the hybrid vesicle treated group showed obviously higher extermination, such as nuclear damage and shrinking of the cells (fragmentized and pyknotic nuclei), in comparison with the control groups.[44] No morphology loss or structural destruction of the cells was found in only vesicle treated mice. The change of body weight was also negligible by various treatments (Figure S18), indicating the photothermal therapy by the hybrid vesicle is biocompatible. 


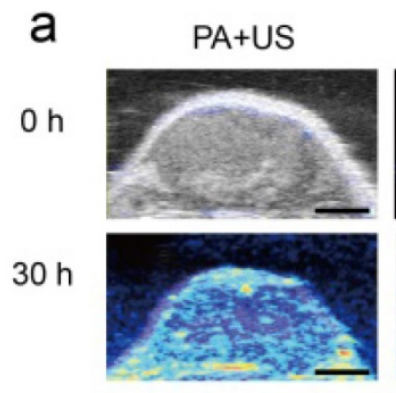

$\mathrm{Oh}$

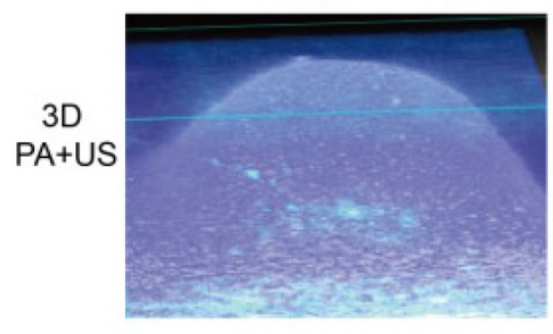

PA
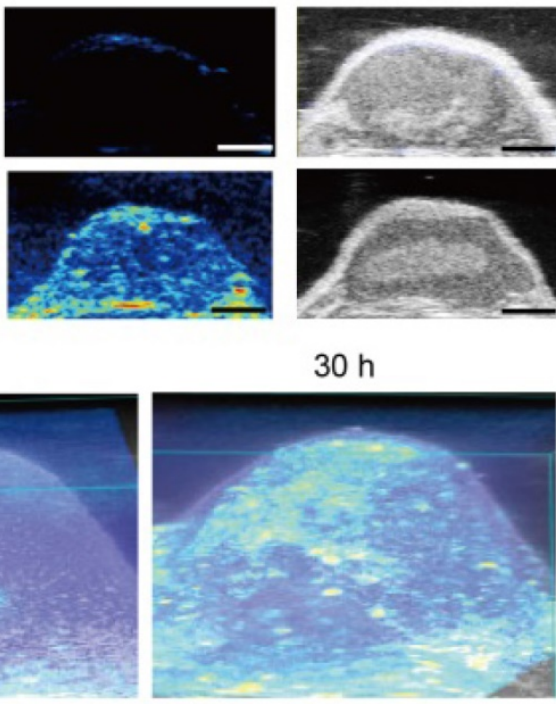

b

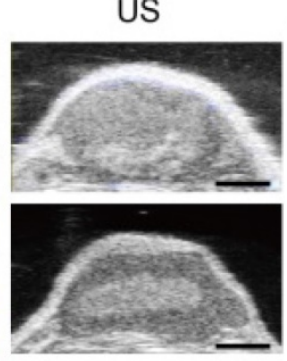

$c$

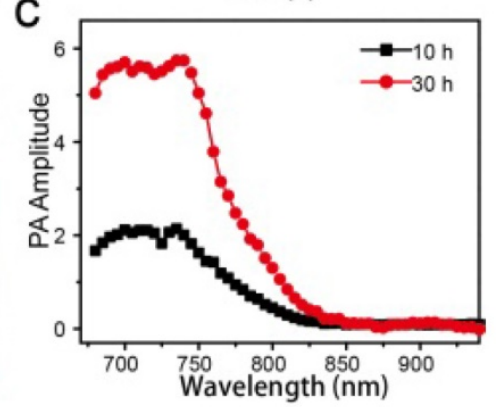

Figure 6. (a) In vivo ultrasound and PA 2D and 3D images before and after intravenous injection of Au@PPDI/PEG vesicle.(Scale bar: 1 mm) (b) PA signal variation in the tumor with time and (c) PA spectra of the vesicle in the tumor region after intravenous injection of the vesicles.

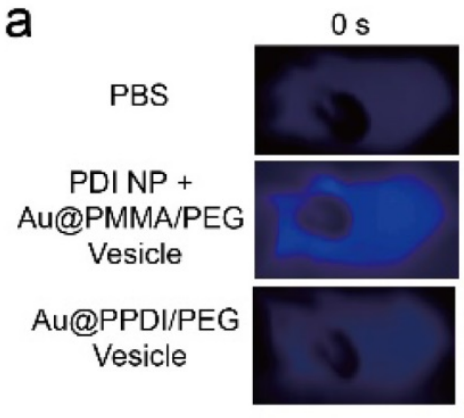

b

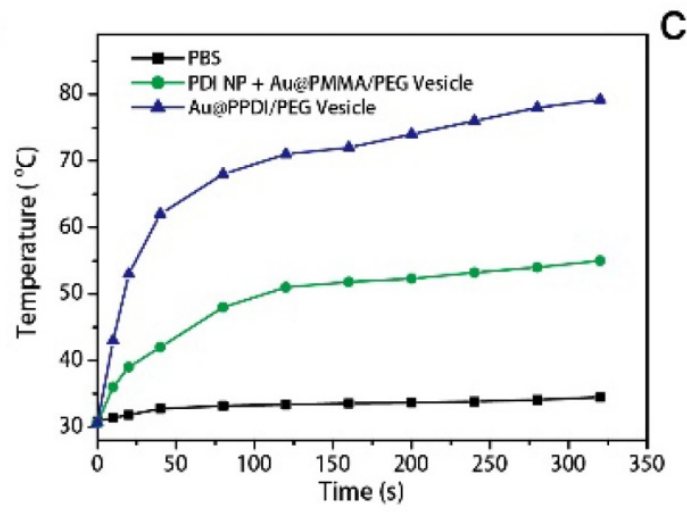

d
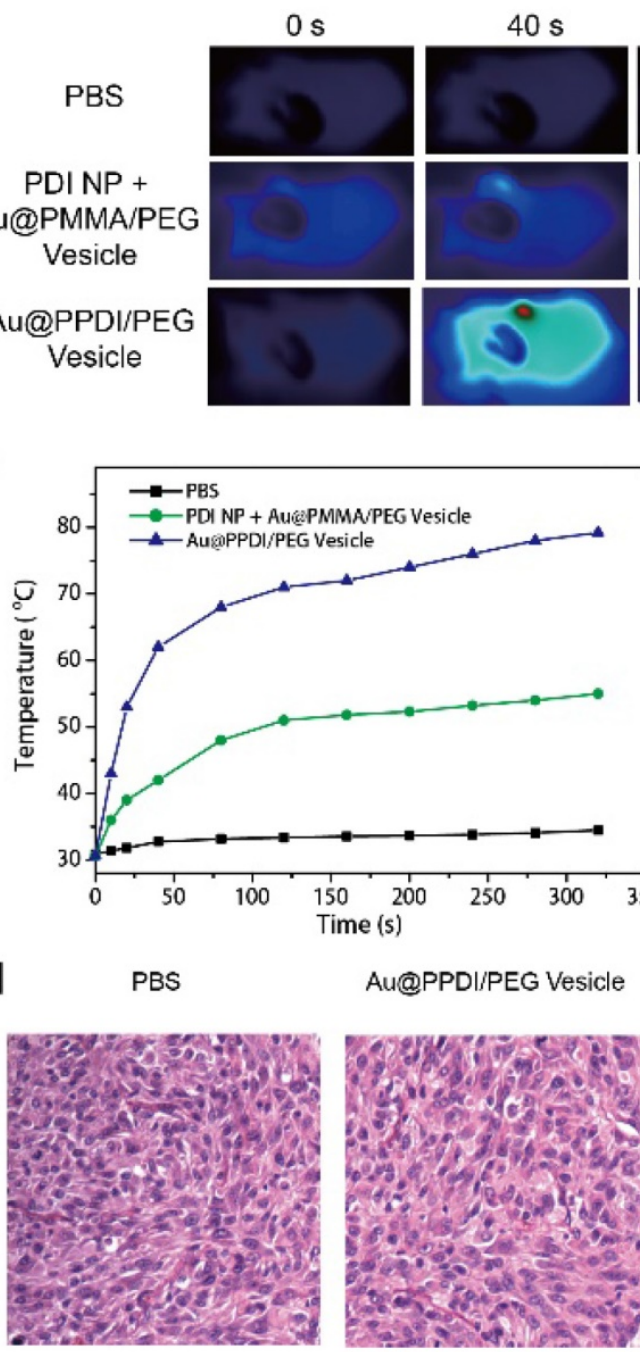

AU@PPDI/PEG Vesicle
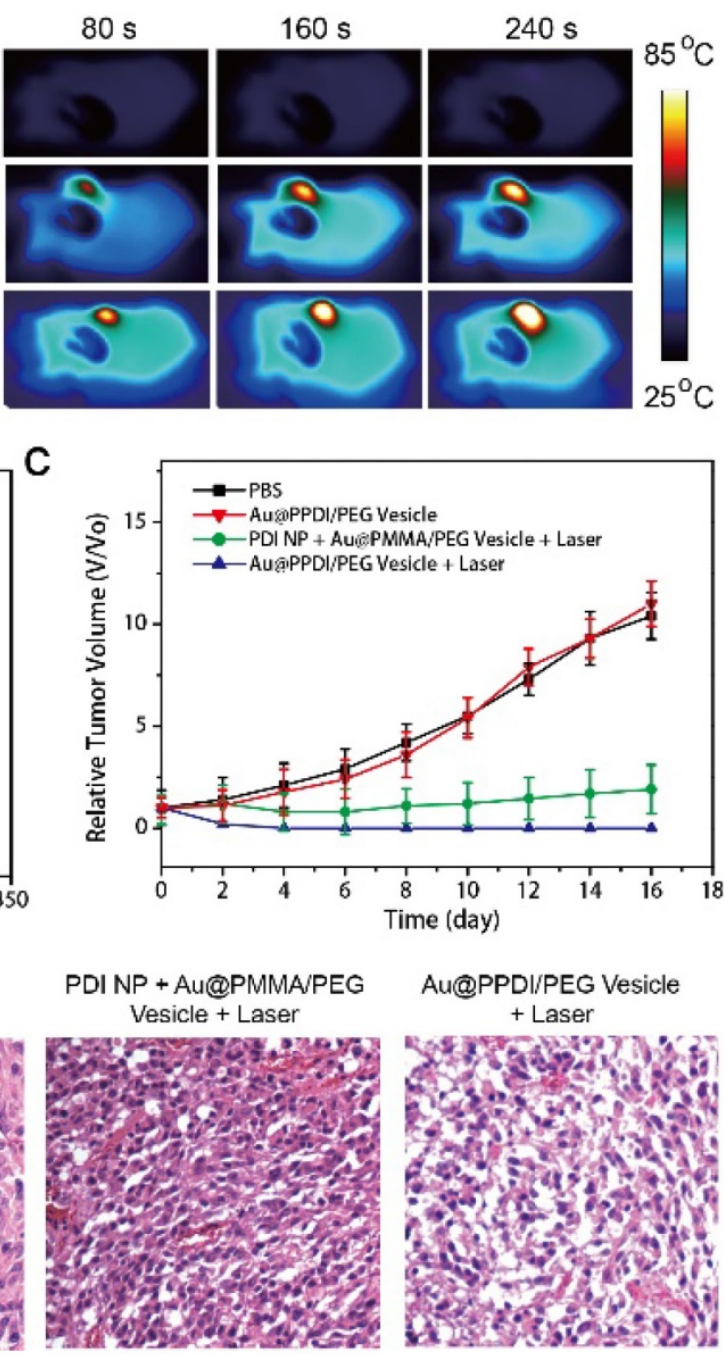

Figure 7. Thermographic images (a) and average temperature variation (b) of the tumor region exposed with NIR laser. Relative tumor growth curves (c) and images of H\&E stained tumor sections (d) of the tumor-bearing mice after injecting the samples intravenously and irradiated with the NIR laser. Tumor volumes were normalized to their initial sizes. 


\section{Conclusions}

In summary, we developed a novel semiconducting-plasmonic Au@PPDI/PEG vesicle with strong NIR absorption and greatly enhanced PA performance. The plasmonic coupling of the AuNPs in the vesicle enhances the light absorption efficiency and PA signal of PPDI. It was demonstrated that the PA signal of the Au@PPDI/PEG vesicle is about 3.5 times higher than that of the simple mixture of PDI NPs and $\mathrm{Au}$ vesicles. The enhanced EM field distribution of the semiconducting-plasmonic vesicle was confirmed by computer simulation. This new type of vesicular assembly demonstrates an original approach to tune the optical interaction between semiconducting polymers and metal nanoparticles. Our results verified that the hybrid vesicle with enhanced photothermal efficiency can serves as excellent PA probe for tumor imaging with high resolution and effective photothermal therapy of cancer.

\section{Supplementary Material}

Synthesis scheme, ${ }^{1} \mathrm{H}$ NMR, ${ }^{13} \mathrm{C}$ NMR, GPC, and

MALDI-TOF mass of the PDI molecules and

polymers, TGA, DLS, Thermographic images and

mice body weight changes.

http://www.thno.org/v07p2177s1.pdf

\section{Acknowledgements}

This work was financially supported by the Intramural Research Program (IRP) of the NIBIB, $\mathrm{NIH}$, the Arkansas Breast Cancer Research Program, the National Natural Science Foundation of China (No. 21674048, 21574064, 61378081), and the Natural Science Foundation of Jiangsu Province of China (No. NY211003, BM2012010) and Innovation Program for Ordinary Higher Education Graduate of Jiangsu Province of China (No. KYLX_0796).

\section{Competing Interests}

The authors have declared that no competing interest exists.

\section{References}

1. Nie L, Chen X. Structural and functional photoacoustic molecular tomography aided by emerging contrast agents. Chem Soc Rev.. 2014;43:7132-70.

2. Cai X, Liu X, Liao L-D, Bandla A, Ling JM, Liu Y-H, et al. Encapsulated Conjugated Oligomer Nanoparticles for Real-Time Photoacoustic Sentinel Lymph Node Imaging and Targeted Photothermal Therapy. Small. 2016;12:4873-80

3. Wang B, Yantsen E, Larson $\mathrm{T}$, Karpiouk AB, Sethuraman $\mathrm{S}$, Su JL, et al. Plasmonic Intravascular Photoacoustic Imaging for Detection of Macrophages in Atherosclerotic Plaques. Nano Lett. 2008:9:2212-7.

4. Pu K, Chattopadhyay N, Rao J. Recent advances of semiconducting polymer nanoparticles in in vivo molecular imaging. J. Control Release. 2016;240:312-22.

5. Miao Q, Pu K. Emerging Designs of Activatable Photoacoustic Probes for Molecular Imaging. Bioconjug Chem. 2016;27:2808-23.
6. Fan Q, Cheng K, Hu X, Ma X, Zhang R, Yang M, et al. Transferring Biomarker into Molecular Probe: Melanin Nanoparticle as a Naturally Active Platform for Multimodality Imaging. J Am Chem Soc. 2014;136:15185-94

7. Zhen X, Zhang C, Xie C, Miao Q, Lim KL, Pu K. Intraparticle Energy Level Alignment of Semiconducting Polymer Nanoparticles to Amplify Chemiluminescence for Ultrasensitive In Vivo Imaging of Reactive Oxygen Species. ACS Nano. 2016;10:6400-9.

8. Xie C, Zhen X, Lei Q, Ni R, Pu K. Self-Assembly of Semiconducting Polymer Amphiphiles for In Vivo Photoacoustic Imaging. Adv Funct Mater. 2017;27:1605397.

9. Fan $\mathrm{Q}$, Cheng $\mathrm{K}$, Yang $\mathrm{Z}$, Zhang $\mathrm{R}$, Yang $\mathrm{M}, \mathrm{Hu} \mathrm{X}$, et al. Photoacoustic Imaging: Perylene-Diimide-Based Nanoparticles as Highly Efficient Photoacoustic Agents for Deep Brain Tumor Imaging in Living Mice. Adv Mater. 2015;27:774.

10. Yang Z, Yuan Y, Jiang R, Fu N, Lu X, Tian C, et al. Homogeneous near-infrared emissive polymeric nanoparticles based on amphiphilic diblock copolymers with perylene diimide and PEG pendants: self-assembly behavior and cellular imaging application. Polym Chem. 2014;5:1372-80.

11. Yang $\mathrm{K}, \mathrm{Xu} \mathrm{H}$, Cheng L, Sun $\mathrm{C}$, Wang J, Liu Z. In Vitro and In Vivo Near-Infrared Photothermal Therapy of Cancer Using Polypyrrole Organic Nanoparticles. Adv Mater. 2012;24:5586-92.

12. $\mathrm{Pu} \mathrm{K}$, Mei J, Jokerst JV, Hong G, Antaris AL, Chattopadhyay N, et al. Diketopyrrolopyrrole-Based Semiconducting Polymer Nanoparticles for In Vivo Photoacoustic Imaging. Adv Mater 2015;27:5184-90.

13. Fan Q, Cheng $\mathrm{K}, \mathrm{Hu} \mathrm{X}, \mathrm{Ma} \mathrm{X}$, Zhang R, Yang M, et al. Transferring Biomarker into Molecular Probe: Melanin Nanoparticle as a Naturally Active Platform for Multimodality Imaging. J Am Chem Soc. 2014;136:15185-94.

14. de la Zerda A, Bodapati S, Teed R, May SY, Tabakman SM, Liu Z, et al. Family of Enhanced Photoacoustic Imaging Agents for High-Sensitivity and Multiplexing Studies in Living Mice. ACS Nano. 2012;6:4694-701.

15. De La Zerda A, Zavaleta C, Keren S, Vaithilingam S, Bodapati S, Liu Z, et al. Carbon nanotubes as photoacoustic molecular imaging agents in living mice. Nat Nanotechnol. 2008;3:557-62.

16. Miao Q, Lyu Y, Ding D, Pu K. Semiconducting Oligomer Nanoparticles as an Activatable Photoacoustic Probe with Amplified Brightness for In Vivo Imaging of $\mathrm{pH}$. Adv Mater. 2016;28:3662-8.

17. Shuhendler AJ, Pu K, Cui L, Uetrecht JP, Rao J. Real-time imaging of oxidative and nitrosative stress in the liver of live animals for drug-toxicity testing. Nat Biotechnol. 2014;32:373-80.

18. Cai Y, Liang $\mathrm{P}$, Tang $\mathrm{Q}$, Yang $\mathrm{X}$, Si W, Huang $\mathrm{W}$, et al. Diketopyrrolopyrrole-Triphenylamine Organic Nanoparticles as Multifunctional Reagents for Photoacoustic Imaging-Guided Photodynamic/Photothermal Synergistic Tumor Therapy. ACS Nano. 2017;11:1054-63.

19. Saha K, Agasti SS, Kim C, Li X, Rotello VM. Gold Nanoparticles in Chemical and Biological Sensing. Chem Rev. 2012;112:2739-79.

20. Halas NJ. Plasmonics: An Emerging Field Fostered by Nano Letters. Nano Lett. 2010;10:3816-22

21. Song J, Duan B, Wang C, Zhou J, Pu L, Fang Z, et al. SERS-Encoded Nanogapped Plasmonic Nanoparticles: Growth of Metallic Nanoshell by Templating Redox-Active Polymer Brushes. J Am Chem Soc. 2014;136:6838-41

22. Kneipp K, Haka AS, Kneipp H, Badizadegan K, Yoshizawa N, Boone C, et al. Surface-Enhanced Raman Spectroscopy in Single Living Cells Using Gold Nanoparticles. Appl Spectrosc. 2002;56:150-4.

23. Karg M, Hellweg T, Mulvaney P. Self-Assembly of Tunable Nanocrystal Superlattices Using Poly-(NIPAM) Spacers. Adv Funct Mater. 2011;21:4668-76.

24. Song J, Yang X, Jacobson O, Huang P, Sun X, Lin L, et al. Ultrasmall Gold Nanorod Vesicles with Enhanced Tumor Accumulation and Fast Excretion from the Body for Cancer Therapy. Adv Mater. 2015;27:4910-7.

25. Li M, Johnson S, Guo HT, Dujardin E, Mann S. A Generalized Mechanism for Ligand-Induced Dipolar Assembly of Plasmonic Gold Nanoparticle Chain Networks. Adv Funct Mater. 2011;21:851-9.

26. Vigderman L, Zubarev ER. Therapeutic platforms based on gold nanoparticles and their covalent conjugates with drug molecules. Adv Drug Deliv Rev. 2013;65:663-76.

27. Huang J, Guo M, Ke H, Zong C, Ren B, Liu G, et al. Rational Design and Synthesis of $\gamma \mathrm{Fe} 2 \mathrm{O} 3 @ \mathrm{Au}$ Magnetic Gold Nanoflowers for Efficient Cancer Theranostics. Adv Mater. 2015;27:5049-56.

28. Li K, Wang K, Qin W, Deng S, Li D, Shi J, et al. DNA-Directed Assembly of Gold Nanohalo for Quantitative Plasmonic Imaging of Single-Particle Catalysis. J Am Chem Soc. 2015;137:4292-5.

29. Lin L-S, Yang $X$, Niu $G$, Song J, Yang $H-H$, Chen $X$. Dual-enhanced photothermal conversion properties of reduced graphene oxide-coated gold superparticles for light-triggered acoustic and thermal theranostics. Nanoscale. 2016;8:2116-22

30. Song J, Wang F, Yang X, Ning B, Harp MG, Culp SH, et al. Gold Nanoparticle Coated Carbon Nanotube Ring with Enhanced Raman Scattering and Photothermal Conversion Property for Theranostic Applications. J Am Chem Soc. 2016;138:7005-15.

31. Song J, Yang X, Jacobson O, Lin L, Huang P, Niu G, et al. Sequential Drug Release and Enhanced Photothermal and Photoacoustic Effect of Hybrid Reduced Graphene Oxide-Loaded Ultrasmall Gold Nanorod Vesicles for Cancer Therapy. ACS Nano. 2015;9:9199-209.

32. Huang $\mathrm{P}$, Lin J, Li W, Rong P, Wang Z, Wang S, et al. Biodegradable Gold Nanovesicles with an Ultrastrong Plasmonic Coupling Effect for 
Photoacoustic Imaging and Photothermal Therapy. Angew Chem Int Ed. 2013;52:13958-64.

33. Darby BL, Auguié B, Meyer M, Pantoja AE, Le Ru EC. Modified optical absorption of molecules on metallic nanoparticles at sub-monolayer coverage. Nat Photonics. 2016;10:40-5.

34. Lim D-K, Barhoumi A, Wylie RG, Reznor G, Langer RS, Kohane DS. Enhanced Photothermal Effect of Plasmonic Nanoparticles Coated with Reduced Graphene Oxide. Nano Lett. 2013;13:4075-9.

35. Moon H, Kumar D, Kim H, Sim C, Chang J-H, Kim J-M, et al. Amplified Photoacoustic Performance and Enhanced Photothermal Stability of Reduced Graphene Oxide Coated Gold Nanorods for Sensitive Photoacoustic Imaging. ACS Nano. 2015;9:2711-9.

36. Song J, Cheng L, Liu A, Yin J, Kuang M, Duan H. Plasmonic Vesicles of Amphiphilic Gold Nanocrystals: Self-Assembly and External-Stimuli-Triggered Destruction. J Am Chem Soc. 2011;133:10760-3.

37. Song J, Huang P, Duan H, Chen X. Plasmonic Vesicles of Amphiphilic Nanocrystals: Optically Active Multifunctional Platform for Cancer Diagnosis and Therapy. Acc Chem Res 2015;48:2506-15.

38. Liu Y, He J, Yang K, Yi C, Liu Y, Nie L, et al. Folding Up of Gold Nanoparticle Strings into Plasmonic Vesicles for Enhanced Photoacoustic Imaging. Angew Chem Int Ed Engl. 2015;127:16035-8.

39. He J, Wei Z, Wang L, Tomova Z, Babu T, Wang C, et al. Hydrodynamically Driven Self-Assembly of Giant Vesicles of Metal Nanoparticles for Remote-Controlled Release. Angew Chem Int Ed Engl. 2013;52:2463-8.

40. Liu D, Zhou F, Li C, Zhang T, Zhang H, Cai W, et al. Plasmonic Colloidosomes with Broadband Absorption Self-Assembled from Monodispersed Gold Nanospheres by Using a Reverse Emulsion System. Angew Chem Int Ed Engl. 2015;54:9596-600.

41. Lim D-K, Jeon K-S, Hwang J-H, Kim H, Kwon S, Suh YD, et al. Highly uniform and reproducible surface-enhanced Raman scattering from DNA-tailorable nanoparticles with 1-nm interior gap. Nat Nanotechnol. 2011;6:452-60.

42. Huynh E, Lovell JF, Helfield BL, Jeon M, Kim C, Goertz DE, et al. Porphyrin shell microbubbles with intrinsic ultrasound and photoacoustic properties. J Am Chem Soc. 2012;134:16464-7.

43. Li H, Zhang P, Smaga LP, Hoffman RA, Chan J. J Photoacoustic Probes for Ratiometric Imaging of Copper(II). J Am Chem Soc. 2015;137:15628-31.

44. Lv G, Guo W, Zhang W, Zhang T, Li S, Chen S, et al. Near-Infrared Emission CuInS/ZnS Quantum Dots: All-in-One Theranostic Nanomedicines with Intrinsic Fluorescence/Photoacoustic Imaging for Tumor Phototherapy. ACS Nano. 2016;10:9637-45 